\title{
L'effet de serre
}

\author{
Ph. Léonard, E. Beeckmans, D. Noul, F. Hermans \\ pleonard@ulb.ac.be, embeeckm@ulb.ac.be, \\ danielnoul@,brutele.be,Francis.Hermans@ulb.ac.be \\ Experimentarium de l'ULB \\ Campus de la Plaine, Bd du Triomphe \\ CP238 \\ 1050 Ixelles
}

RESUME : Après une description générale de l'effet de serre climatique, on trouvera ci-dessous quelques démonstrations de physique illustrant l'évolution du climat, de la température de la Terre et le rôle du dioxyde de carbone.

Mots clés : expérience démonstrative, effet de serre, albédo, convection, infrarouge, climat.

\section{INTRODUCTION}

Pour décrire la position d'un lieu à la surface de la Terre, les Grecs définirent les « climats » (du grec klima $=$ inclinaison ) c'est-à-dire des régions de mêmes latitudes où la hauteur du Soleil sur l'horizon est identique.

Si une faible latitude est sans doute prometteuse d'un climat assez chaud, nous savons tous combien les changements climatiques sont à l'ordre du jour. Un réchauffement de $0,8^{\circ} \mathrm{C}$ a été observé pendant le seul XXe siècle et il semble prouvé qu'il est lié aux activités humaines au travers d'une augmentation de la concentration de certains gaz d'origine anthropique dans l'atmosphère. Comme cette tendance pourrait se poursuivre dans le futur, c'est un sujet de préoccupation sérieux pour nos sociétés puisque le réchauffement global pourrait atteindre $+3,5^{\circ} \mathrm{C}$ en moyenne d'ici la fin de ce siècle.

Le physicien français Joseph Fourier, qui fut l'un des tout premiers à évoquer l'effet de serre, a établi au début du XIXe siècle les conditions de l'équilibre thermique : lorsque les gains et les pertes en énergie d'un corps se compensent, sa température reste constante et celle-ci est d'autant plus élevée que les gains (et les pertes) le sont.

Pour la Terre, les gains ont le Soleil pour origine, les pertes se font via l'énergie rayonnée vers l'espace. Notre planète reçoit du Soleil un rayonnement incident moyen de $342 \mathrm{~W} / \mathrm{m}^{2}$ (en tenant compte de l'alternance jour/nuit et des différences de latitude) dont environ $50 \%$ sont absorbés par le sol et les océans puis transformés en chaleur.

\section{DESCRIPTION DES PHENOMENES MIS EN EVIDENCE}

\subsection{Albédo}

L'énergie solaire absorbée par une surface dépend des propriétés optiques de cette surface puisqu'une partie $\mathrm{du}$ rayonnement solaire incident est immédiatement réfléchie. La fraction entre le rayonnement solaire réfléchi et le rayonnement solaire incident est appelée coefficient de réflexion ou albédo.

$\begin{array}{ll} & \text { Albédo } \\ \text { Neige } & 0,75 \text { à } 0,95 \\ \text { Désert } & 0,3 \\ \text { Forêt } & 0,1 \\ \text { Océan } & 0,05 \\ \text { Peinture blanche } & 0,8 \\ \text { Peinture noire } & 0,05 \\ \text { Planète Terre } & 0,3 \\ \text { Sable } & 0,18 \text { à } 0,28\end{array}$

Il est clair que si l'albédo de notre planète augmente, sa température aura tendance à diminuer. Or une partie de ce que nous appelons la pollution (notamment certains gaz dits à effet de serre) et les aérosols (brouillard de particules solides ou liquides dont la taille est inférieure au micromètre) favorisent la formation des nuages et les rendent plus brillants (effet appelé "parasol") : il s'agit là d'un forçage négatif des activités anthropiques sur le climat. Par contre les dépôts de suie et la diminution des surfaces glacées (brillantes) ont un résultat inverse : c'est un forçage positif.

\subsection{Convection}

L'énergie solaire absorbée par la surface de la Terre est transmise à l'atmosphère principalement par convection et par les cycles d'évaporation et de condensation de l'eau, puis émise par l'atmosphère vers l'espace sous forme de rayonnement INFRAROUGE (IR).

La convection est le transport de la chaleur par des mouvements au sein d'un fluide. En effet, des différences de température produisent des différences de masses volumiques (par dilatation) et ainsi des mouvements relatifs des parties d'un fluide à températures différentes. Dans une serre horticole, c'est principalement cet échange thermique entre l'intérieur et l'extérieur que l'on réduit de façon à créer en espace protégé du vent (qui renforce la convection) et des basses températures. Certains expliquent le fonctionnement d'une serre en se basant sur le fait que le verre n'est pas transparent dans l'IR (vers $10 \mu \mathrm{m}$ ). Or une serre construite à l'aide de polyéthylène, transparent dans l'IR, est presque aussi efficace qu'une serre en verre... 


\subsection{Rayonnement infrarouge}

Le rayonnement INFRAROUGE était appelé "chaleur obscure" au temps de Fourier : il transporte de l'énergie mais on le ne voit pas. Tout corps émet un rayonnement dont la puissance totale et le spectre dépendent de sa température. On peut donc affirmer que le rayonnement thermique trahit la température d'un objet. Le spectre désigne la répartition de la puissance dans les différentes longueurs d'onde (c'est-à-dire la couleur, dans la gamme du visible).

Les lois de Stefan et de Wien stipulent qu'un corps chaud rayonne une puissance proportionnelle à T4 (en $\mathrm{K}$ ) et que cette énergie répartie sur toutes les longueurs d'onde présente un maximum pour une longueur d'onde particulière $\lambda$ qui est telle que T. $\lambda=2896 \mu \mathrm{m} . \mathrm{K}$

Le rayonnement solaire, par exemple, est émis par sa surface à $5500{ }^{\circ} \mathrm{C}$ environ (comme un corps noir à $5777 \mathrm{~K}$ ) et, à cette température, $40 \%$ de l'énergie émise est visible (de $0,4 \mu \mathrm{m}$ - violet bleu - à $0,7 \mu \mathrm{m}$-rouge, avec un max vers $0,5 \mu \mathrm{m}$ dans le jaune). $50 \%$ de cette énergie nous reste invisible mais est capable de réchauffer un thermomètre qui y est exposé : c'est un rayonnement infrarouge (sa longueur d'onde est plus grande que celle du rouge, jusqu'à $3 \mu \mathrm{m}=$ infrarouge proche). Les $10 \%$ restants sont des ultraviolets.

Pour des objets à température usuelle et donc beaucoup plus faible, la plus grande partie du rayonnement est émise dans l'infrarouge lointain (de $3 \mu \mathrm{m}$ à $50 \mu \mathrm{m}$ ). Dans l'obscurité, à température normale, ni les hommes ni les animaux ne sont visibles puisque le rayonnement qu'ils émettent ne l'est pas ! Il faudrait atteindre les $700^{\circ} \mathrm{C}$ au moins pour qu'une petite partie du rayonnement émis soit discernable ; c'est la cas de la lave des volcans (rouge sombre).

Certains matériaux réagissent très différemment à ces deux gammes de rayonnement (visible et IR). Ainsi le verre, le plexiglas, les polycarbonates, la vapeur d'eau, le méthane et le tristement célèbre dioxyde de carbone sont transparents pour la lumière visible mais ils absorbent les IR.

C'est au chimiste et ingénieur irlandais John Tyndall que l'on doit les premières données expérimentales sur l'absorption et l'émission des IR par les gaz.

Une partie du rayonnement solaire traverse l'atmosphère puis est partiellement absorbée par le sol qui chauffe et réémet vers l'espace un rayonnement infrarouge (lointain) correspondant à sa température. Si la puissance absorbée est environ $200 \mathrm{~W} / \mathrm{m}^{2}$, celle qui est réémise est $200 \mathrm{~W} / \mathrm{m}^{2}$ et la température reste constante. $\mathrm{Si}$, par la présence des gaz à effet de serre, l'atmosphère terrestre devient opaque aux infrarouges lointains, elle les absorbe, chauffe davantage, et rayonne des IR dans toutes les directions. Vers l'espace, la puissance rayonnée est toujours $200 \mathrm{~W} / \mathrm{m} 2$ puisque sa température s'est stabilisée. Par contre l'atmosphère rayonne aussi vers le sol : une même puissance de 200, par symétrie. C'est donc qu'elle a reçu du sol une puissance de $400 \mathrm{~W} / \mathrm{m}^{2}$ ! Celui-ci a vu sa température augmenter considérablement : c'est l'effet de serre. Remarquez que le sol a lui aussi trouvé une situation d'équilibre à température plus élevée : il reçoit 200 du Soleil, 200 de l'atmosphère et rayonne 400 .

Nous bénéficions heureusement d'un effet de serre naturel (nuages, vapeur d'eau) qui permet à la Terre d'avoir une température de $30^{\circ} \mathrm{C}$ supérieure à celle qu'elle aurait sans atmosphère $\left(15^{\circ} \mathrm{C}\right.$ de moyenne au lieu de $-18^{\circ} \mathrm{C}$ ).

L'augmentation de la concentration des gaz à effet de serre suite aux activités humaines (combustions, déforestation, pollution) déplace l'équilibre vers des températures plus élevées : il y a donc piégeage d'une partie de la puissance solaire pendant un temps qui correspond au changement de température. Actuellement le déséquilibre s'élève environ à $1 \mathrm{~W} / \mathrm{m}^{2}$.

Par ciel clair, la vapeur d'eau est responsable de $60 \%$ de l'effet de serre $(0,3 \%$ de la composition atmosphérique), et le dioxyde de carbone de $26 \%(0,0383 \%$ de l'atmosphère terrestre), le méthane ( $\mathrm{CH} 4: 1,8 \mathrm{ppmv})$, le protoxyde d'azote (N2O : 0,3 ppmv), l'ozone (O3 : 0,03 ppmv) et d'autres gaz étant responsables des $14 \%$ restants.

La liste suivante donne l'état des choses en termes des forçages climatiques :

$\begin{array}{ll}\mathrm{CO}_{2} & 1,4+/-0,2 \mathrm{~W} / \mathrm{m}^{2} \\ \mathrm{CH}_{4} & 0,7+/-0,2 \\ \mathrm{O}_{3} & 0,3+/-0,15 \\ \mathrm{~N}_{2} \mathrm{O} & 0,15+/-0,05 \\ \mathrm{CFC} & \text { En pos }: 0,35+/-0,05 \\ & \text { En nég }:-0,1+/-0,05 \\ \text { aérosols atm } & -0,4+/-0,3 \mathrm{~W} / \mathrm{m}^{2} \\ \text { aérosols et nuages } & -1+/-0,5 \\ \text { albédo continental } & -0,2+/-0,2 \\ \text { Soleil } & +0,4+/-0,2 \\ \text { volcans } & \text { Entre }+0,2 \text { et }-0,5\end{array}$

\section{DEMONSTRATIONS}

\subsection{Visualisation de la convection}

Dans un aquarium rempli d'eau claire (Fig. 1), on laisse tomber quelques gouttes de colorant alimentaire dont la densité est plus grande que celle de l'eau. L'aquarium est chauffé du côté droit par une lampe et des glaçons refroidissent l'eau du côté gauche. Un large mouvement de convection emmène le colorant dans une rotation en sens inverse des aiguilles d'une montre.

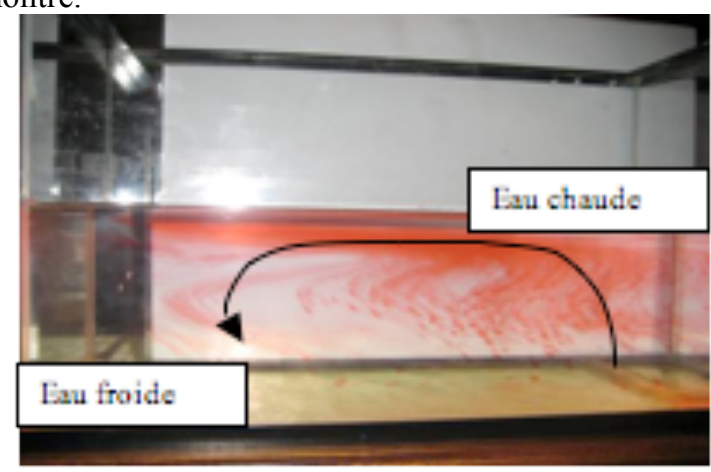

Fig 1 : Visualisation de la convection dans un liquide 


\subsection{Albédo}

Deux plaques identiques (Fig. 2) si ce n'est leur couleur - l'une est noire, l'autre métallisée - sont placées à la même distance d'une lampe. La température de la plaque noire s'élève beaucoup plus que celle de la plaque métallisée, dans le même temps.

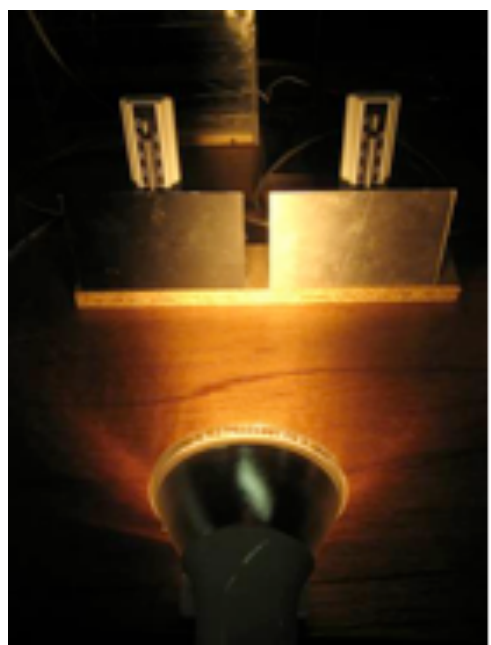

Fig 2 : Deux plaques d'albédo différent

\subsection{Transmission des infrarouges à travers les ma- tériaux}

Il est intéressant de tester les matériaux suivant leur absorption des IR. Le montage suivant (Fig. 3) le permet.

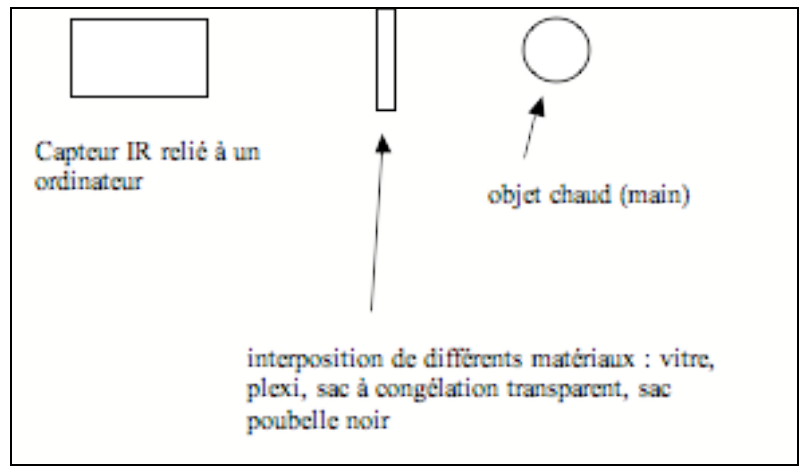

Fig 3: Mesure de la transparence aux infrarouges

La vitre et le plexi arrêtent les IR et sont transparents au visible. Le sachet de congélation est transparent à la fois pour le visible et les IR tandis que le sac poubelle noir laisse passer les IR mais pas le visible.

\subsection{Effet de serre "horticole"}

Deux boîtes identiques (Fig. 4) sont placées devant deux lampes de 120W. Une des boîtes est fermée par une vitre, l'autre par une feuille de cellophane. La température initiale des deux boîtes est $20^{\circ} \mathrm{C}$. Après une dizaine de minutes, les températures sont toujours à peu près identiques $\left(70^{\circ} \mathrm{C}\right.$ pour le verre et $67^{\circ} \mathrm{C}$ pour le cellophane). Dans une serre, l'effet principal est donc bien le blocage de la convection.

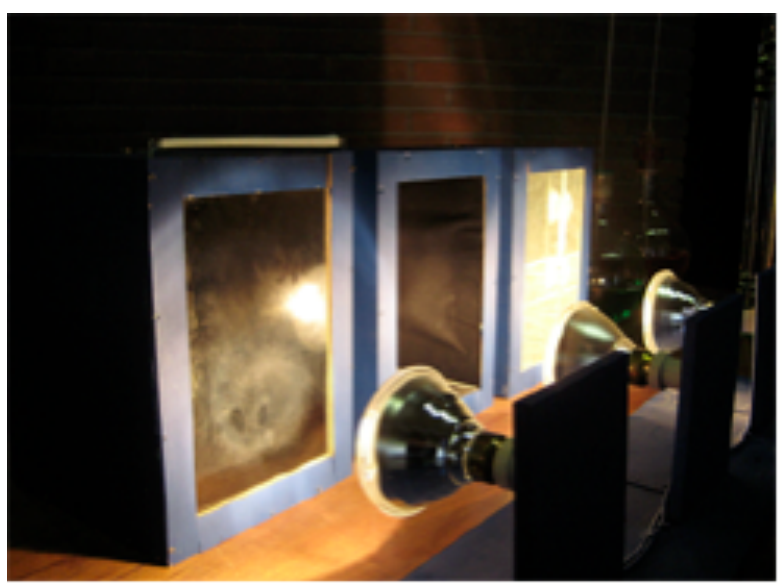

Fig 4 : Effet de serre

\subsection{Effet de serre climatique.}

Expérience (Fig. 5) montrant l'effet d'une augmentation de la concentration en $\mathrm{CO}_{2}$ dans l'atmosphère au-dessus d'une surface en équilibre thermique.

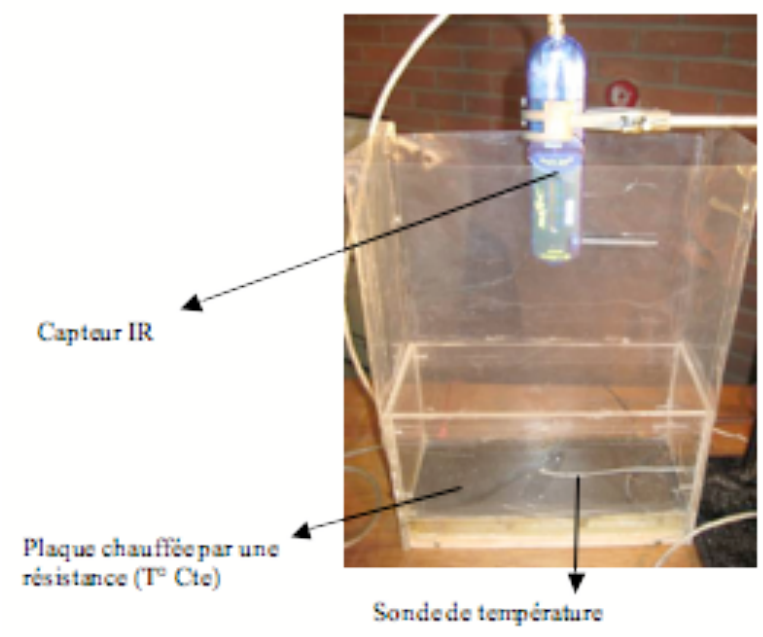

Fig. 5 : Effet de serre dî au $\mathrm{CO}_{2}$

Une plaque métallique, jouant le rôle de la surface terrestre, se trouve au fond d'un bac (un aquarium sans eau). L'air se trouvant dans le récipient au-dessus de la plaque correspond aux basses couches de l'atmosphère. Un détecteur de température, collé à la plaque, permet de suivre la température de celle-ci. Un détecteur de rayonnement infrarouge est placé au dessus du bac et permet de mesurer l'énergie rayonnée par la plaque. Une résistance chauffante située en dessous de la plaque permet d'apporter une quantité d'énergie constante à cette plaque. En réponse à cet apport, la plaque va se réchauffer. De ce fait, elle va dégager de plus en plus de chaleur par convection et par rayonnement infrarouge. Elle finira par atteindre une température constante, température qui correspond à l'équilibre entre 

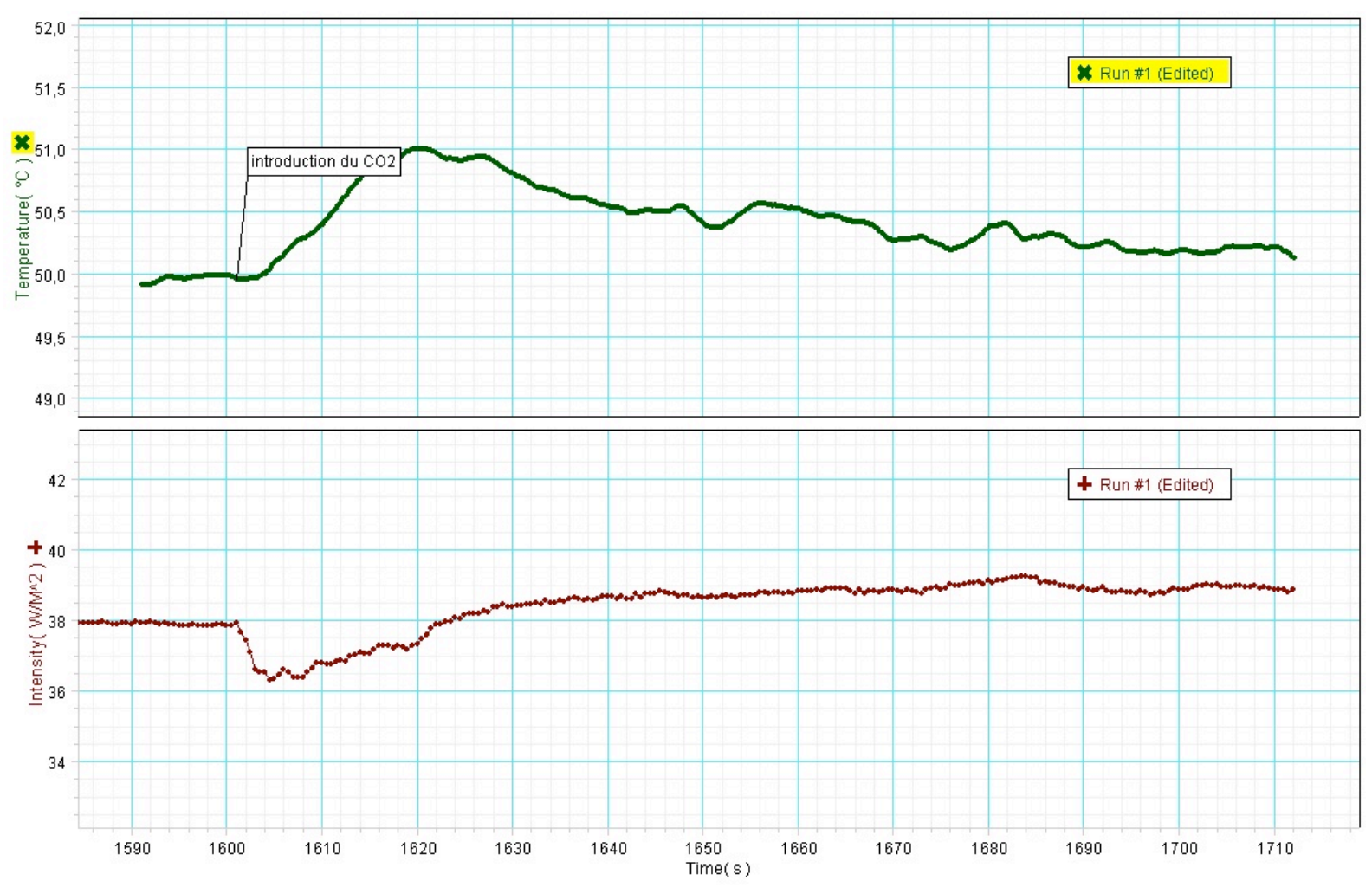

l'apport d'énergie et les pertes. Nous sommes dans les mêmes conditions que la surface terrestre puisque les rayons du soleil, qui ont traversé les hautes couches de l'atmosphère, sont essentiellement absorbés par la surface terrestre et non par la troposphère (basse couche de l'atmosphère, siège des phénomènes climatiques). À un certain moment, on introduit, doucement, du $\mathrm{CO}_{2}$ dans l'air du bac. L'effet d'écran du $\mathrm{CO}_{2}$ vis-à-vis du rayonnement infrarouge est immédiat et se traduit par une diminution du rayonnement au niveau du détecteur. C'est avec un léger retard que la température de la plaque augmente suite à cette augmentation de l'effet de serre. $\mathrm{Le} \mathrm{CO}_{2}$ qui a été injecté finit par s'en aller par convection. Le rayonnement infrarouge détecté remonte et la température redescend très lentement vers sa valeur précédant l'introduction de $\mathrm{CO}_{2}$.

Les graphiques ci-dessus, représentent un enregistrement type de l'expérience. La courbe du haut donne l'évolution de la température en fonction du temps. Le graphique du bas représente l'évolution de l'intensité du rayonnement infrarouge.

\section{Matériel utilisé :}

- capteur IR PS-2148 Pasco (réponse uniforme entre 580 et $40000 \mathrm{~nm}$ )

- capteur de température sur PS-2170 Pasco ($35^{\circ} \mathrm{C}$ to $135^{\circ} \mathrm{C},+/-0,5^{\circ} \mathrm{C}$ )

- $\quad$ siphon pour injecter le $\mathrm{CO}_{2}$ (en capsules)

- ordinateur avec logiciel Datastudio (Pasco)

- récipient muni d'une plaque chauffante.

\section{CONCLUSION}

La simulation de l'effet de serre climatique implique d'observer la coïncidence de l'absorption du rayonnement infrarouge par l'atmosphère du modèle et d'une montée en température de sa surface. Le dispositif proposé ici remplit parfaitement cet objectif.

Une démonstration critique a également permis de confirmer le rôle essentiel joué par la convection dans la serre horticole et généralement attribué, à tort, aux infrarouges.

\section{Bibliographie}

* Evaluation of the Planck-mean absorption coefficients from HITRAN and HITEMP databases, par H. Zhang et M. F. Modest, Journal of Quantitative Spectroscopy \& Radiative Transfer 73 (2002) 649-653

* Aéronomie par G. Kockarts, De Boeck Université.

* Renewable energy, edited by G. Boyle. Oxford University.

* I.Catala et al. (sous la dir. de), Graines de sciences 2, Le Pommier, 2000.

* Effet de serre contre effet de serre, Idées de physique par JM Courty et E Kierlik, Pour La Science.

* Réchauffement global : une bombe à retardement? par J. Hansen, Pour La Science N`318 avril 2004.

* Exploring the greenhouse effect through physics-oriented activities par K.P. Browne et P. W. Laws, Phys Educ. $\mathrm{n}^{\circ} 38$.

* Le transport de la chaleur par rayonnement par J-C Dehaes, Physique Générale ( $3^{\text {ème }}$ partie, Chap. 2.2), Presses universitaires de Bruxelles, INGE1, PHYS-D-101

* Météorologie par Y. Corboz, Belin-Pour La Science.

* Introduction à la climatologie par Huffy, De Boeck.

www.larecherche.fr/special/web/webhs17p1.html www.ipcc.ch

www.effet-de-serre.gouv.fr 